\title{
Sudden unexpected death after acute symptomatic seizures in a patient on mechanical ventilation
}

\author{
Raisa C. Martinez ${ }^{1}$, Naoum P. Issa', Shasha Wu¹, Xi Liu², Sandra Rose ${ }^{1}$ and James X. Tao ${ }^{1 *}$
}

\begin{abstract}
Background: The mechanism of sudden unexpected death in epilepsy remains poorly understood. Seizure induced cardiac arrhythmia, central and obstructive apneas have been proposed as possible causes of death. Here we report a unique case of seizure related sudden unexpected death in a patient whose airway was fully protected by intubation and mechanic ventilation in the absence of fatal cardiac arrhythmia.

Case presentation: A 70-year-old woman was undergoing mechanical ventilation and videoelectroencephalography (EEG) monitoring following two convulsive seizures with ictal hypoventilation and hypoxemia. Several hours after intubation, she suffered another generalized tonic clonic seizure lasted for 3 min and developed postictal generalized EEG suppression in the presence of stable vital signs with $\mathrm{SpO}_{2}>90 \%$. EEG suppression persisted throughout the postictal phase. There was a significant fluctuation of systolic blood pressure between 50 and $180 \mathrm{mmHg}$ with several bouts of hypotension $<60 \mathrm{mmHg}$. She remained unresponsive after the convulsive seizure and died of diffuse cerebral edema $12 \mathrm{~h}$ later. Autopsy revealed no clear cause of death, except for possible hypoxic and ischemic injury leading to the diffuse cerebral edema.
\end{abstract}

Conclusion: Given the reliable periictal airway protection, neither seizure induced central apnea nor obstructive apnea appeared to be the direct cause of death in this unique case. In the absence of fatal cardiac arrhythmia, diffuse cerebral edema secondary to seizure-induced autonomic dysfunction, hypotension and hypoxemia might be the cause of death, highlighting the etiological heterogeneity of sudden unexpected death in epilepsy.

Keywords: Convulsive seizure, SUDEP, Mechanic ventilation, Apnea, Prone position

\section{Background}

Sudden unexpected death in epilepsy (SUDEP) is the leading cause of premature death in patients with chronic refractory epilepsy [1]. Over the last several decades, several risk factors for SUDEP have been proposed including chronic uncontrolled epilepsy, the duration of epilepsy, young age, male sex and intellectual disability [2-4]. Pooled data indicated that the frequency of generalized tonic clonic seizures (GTCS) is the most important risk factor for SUDEP [5]. Lack of night-time

\footnotetext{
*Correspondence: jtao@neurology.bsd.uchicago.edu

'Adult Epilepsy Center, Department of Neurology, The University of Chicago, 5841 South Maryland Ave. MC2030, Chicago, IL 60637, USA

Full list of author information is available at the end of the article
}

supervision and absence of nocturnal listening device are also important risk factors [6]. Nevertheless, the mechanisms of SUDEP have remained poorly understood. SUDEP commonly occurs during sleep and in bed with most cases being unwitnessed [7]. In a minority of witnessed SUDEP cases, cardiorespiratory functions were not adequately monitored, particularly lack of oxygen and respiratory monitoring [8]. Here, we reported a patient with new onset of acute symptomatic seizures who died after a GTCS during videoelectroencephalography (EEG) monitoring, while being mechanically ventilated, which might provide new insights into the mechanisms of SUDEP.

(c) The Author(s). 2020 Open Access This article is licensed under a Creative Commons Attribution 4.0 International License, which permits use, sharing, adaptation, distribution and reproduction in any medium or format, as long as you give appropriate credit to the original author(s) and the source, provide a link to the Creative Commons licence, and indicate if changes were made. The images or other third party material in this article are included in the article's Creative Commons licence, unless indicated otherwise in a credit line to the material. If material is not included in the article's Creative Commons licence and your intended use is not permitted by statutory regulation or exceeds the permitted use, you will need to obtain permission directly from the copyright holder. To view a copy of this licence, visit http://creativecommons.org/licenses/by/4.0/. 


\section{Case presentation}

The patient is a 70-year-old female with a medical history of diabetes mellitus type 2 , hypertension, hyperlipidemia, and herpes zoster of right face who presented with subacute left chest pain, shortness of breath and a diffuse painful rash for 7 days with progressive worsening despite the treatment with oral prednisone and antibiotics. Vital signs upon presentation were normal except for mild tachypnea. Diagnostic tests for pulmonary embolism and acute coronary syndrome were unrevealing. On day 2 of admission a progressive mental status change was noted. Initial head CT was normal. An extended EEG was remarkable for diffuse slowing of background to $5-7 \mathrm{~Hz}$ which was thought to be related to cefepime neurotoxicity. Cefepime was subsequently transitioned to ceftriaxone. In the following days, she continued to have a fluctuating level of awareness, but was in stable condition with an EEG background $7-8 \mathrm{~Hz}$ during a routine EEG study. Brain MRI (3 T) with and without contrast was unremarkable except for two punctate subcortical infarcts.

On day 9 of hospitalization, she had an acute episode of altered mental status, desaturation $\left(\mathrm{SpO}_{2} 80 \%\right)$ with atrial fibrillation (HR 160/min) and bowel incontinence. After vital signs were stabilized by a rapid response team, an EEG was ordered due to suspicion of a seizure. During the hook-up, the patient was noted to have right gaze deviation with left arm and leg clonic jerking. Since the electrodes had not yet been fully applied, EEG was not interpretable during this period. She underwent immediate endotracheal intubation and mechanical ventilation and she was treated with fosphenytoin. Overnight continuous video-EEG captured another generalized tonic clonic seizure that lasted for 3 min while receiving nursing care, which was followed by postictal generalized EEG suppression (PGES) that lasted for approximately 2 min (Fig. 1 ).

After this point, EEG suppression was persisted with rare (1-2 s) bursts of diffuse delta activity. Four subclinical
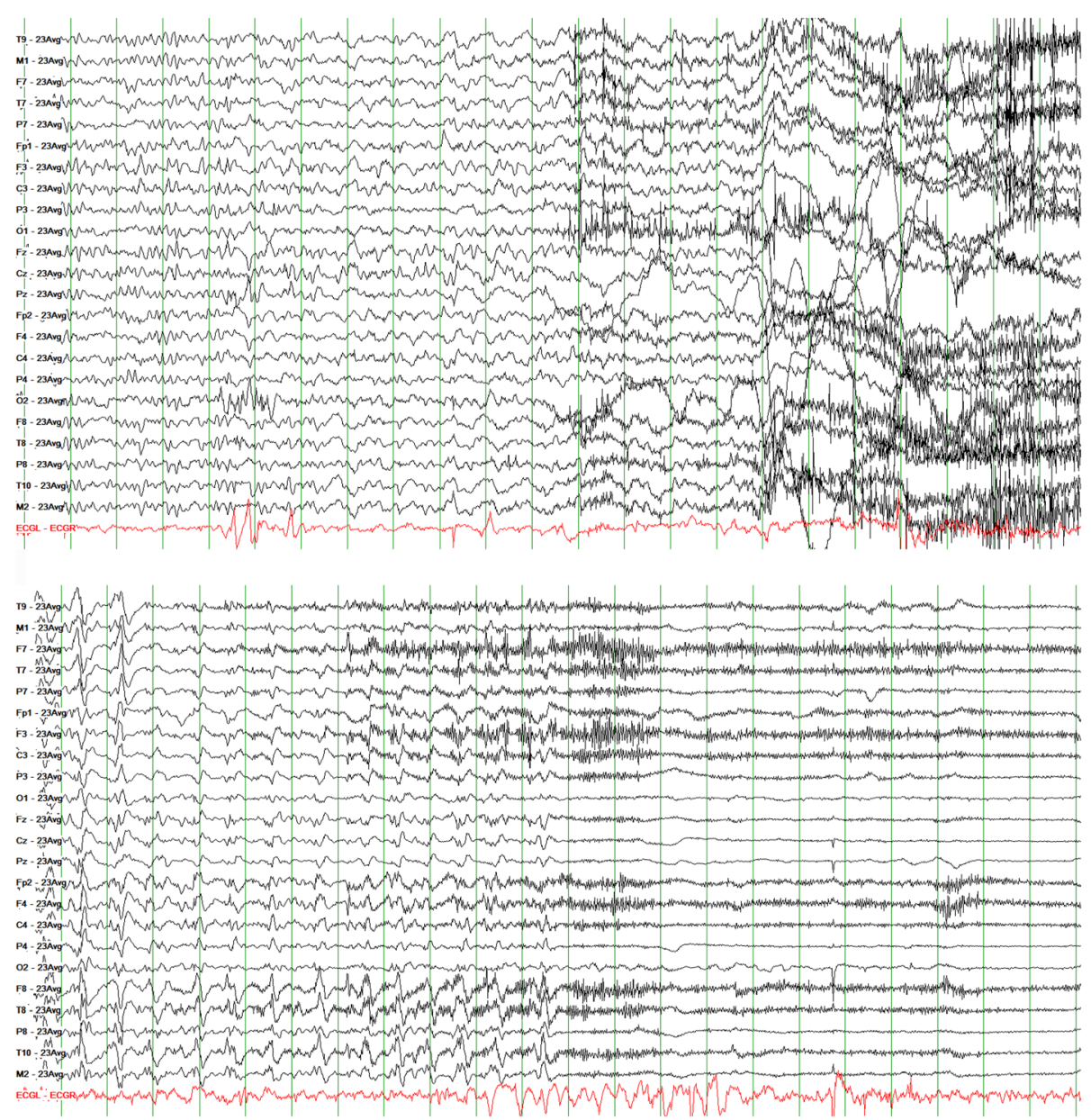

Fig. 1 Top panel showed 7-8 Hz EEG background and unclear ictal onset that was obscured by diffuse muscle artifacts. Bottom panel showed ictal offset and postictal generalized EEG suppression that occurred in the presence of stable vital signs including heart rate, blood pressure and oxygen saturation as shown in Fig. 3. EEG recording settings: high pass filter $1 \mathrm{~Hz}$ and low pass filter $50 \mathrm{~Hz}$. Sensitivity: $10 \mu \mathrm{V} / \mathrm{mm}$ 
nonconvulsive seizures (NCS) were observed over the right hemisphere lasting 30-75 s, and the last NCS was approximately $8 \mathrm{~h}$ after the GTCS. Portable chest X-ray showed possible aspiration pneumonia or mild neurogenic pulmonary edema (Fig. 2a to d). A repeat head CT scans $9 \mathrm{~h}$ after the GTCS did not show acute changes (Fig. 2e and f). After the GTCS, heart rate ranged between 70 and $100 \mathrm{bpm}$ and $\mathrm{O}_{2}$ Sat was $>96 \%$. There was a significant fluctuation of systolic blood pressure between 50 and 180 $\mathrm{mmHg}$ with several bouts of hypotension $<60 \mathrm{mmHg}$ (Fig. 3). Twelve hours later, the EEG became electrically silent and the patient developed dilated, unreactive pupils and absent brainstem reflexes, consistent with brain death. A repeat head CT showed diffuse cerebral edema and loss of gray-white differentiation (Fig. $2 \mathrm{~g}$ ). The patient subsequently died on the same day.

There was no significant hypoxemia, electrolyte imbalance and sepsis prior to the onset of last GTCS. Basic metabolic panel showed sodium 139 and potassium 5.0, chloride 107, anion gap 14, BUN 33, creatinine 1.5 and GFR 34. Complete blood count (WBC) was within normal range except for mild thrombocytopenia (WBC
10.9, RBC 4.6, HB 12.2 and platelet 125). Blood and urine cultures were negative for bacteria. Lactic acid was 2.1. Arterial blood gas showed $\mathrm{PH} 7.43, \mathrm{PCO}_{2} 32, \mathrm{SO}_{2}$ 99.4\%. After the onset of last GTCS, the patient developed significant metabolic acidosis with $\mathrm{PH}$ 7.1, lactic acid 7.9 and worsening renal insufficiency.

Gross examination of the brain during the autopsy demonstrated a somewhat dusky cerebral surface and moderate symmetrical edema. The cerebellar tonsils showed some notching, raising the possibility of tonsillar herniation through the foramen magnum. Microscopic examination revealed shrunken neurons and mottling of the cerebral cortex, suggestive of hypoxic ischemic injury. The meninges contained small foci of acute inflammation. These could be secondary to hypoxic ischemic injury or could represent incipient meningitis. Overall though, the inflammation is interpreted as too subtle and potentially too early to explain the clinically observed cerebral edema. There are no other changes that would explain cerebral edema, and no other inflammatory changes or neoplastic infiltrates are seen. With that, the possibility of hypoxic ischemic brain injury leading to cerebral edema should be considered.
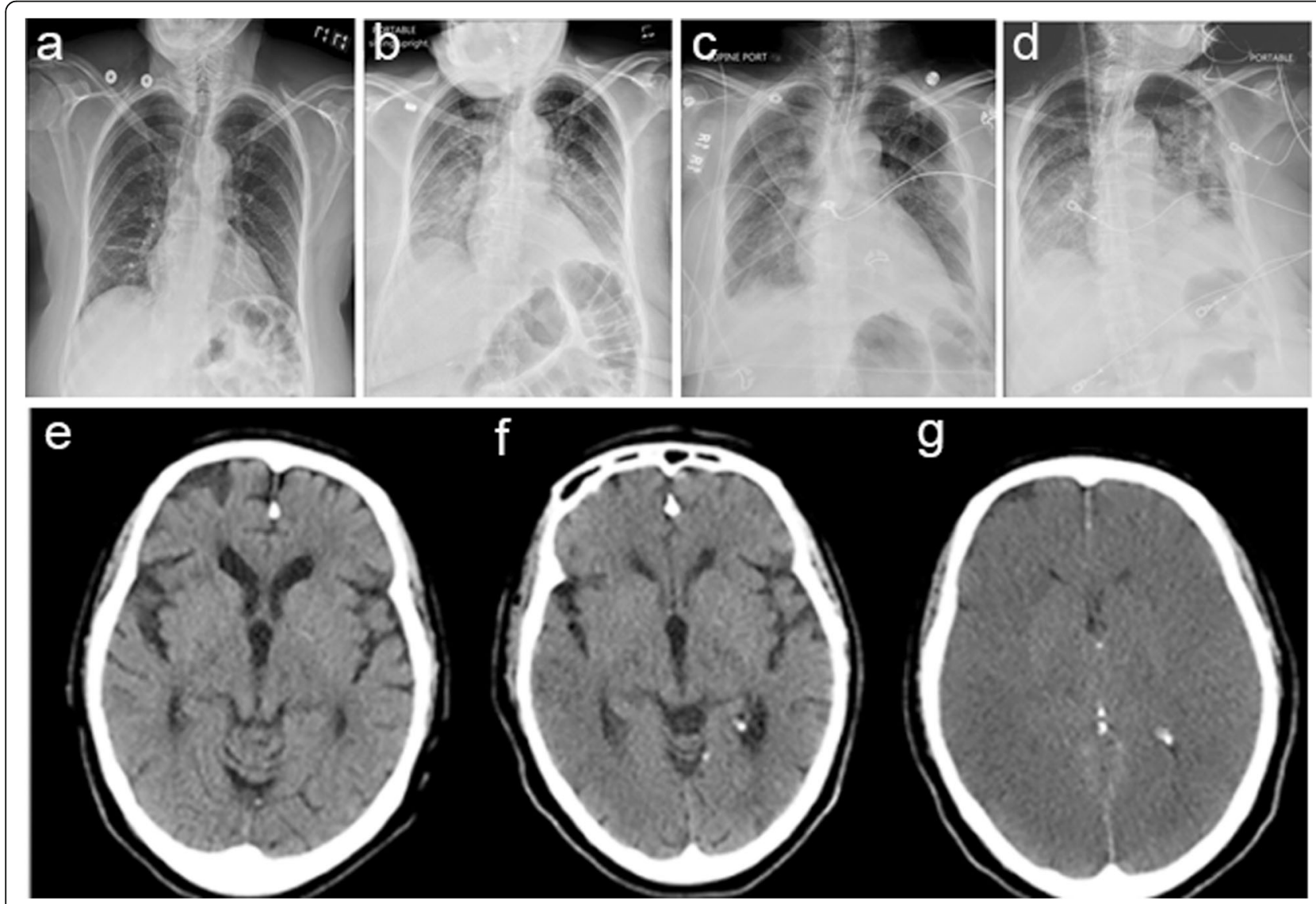

Fig. 2 Periictal chest $x$-ray (CXR) and head CT (HCT) scan: a CXR on admission; b CXR $10 \mathrm{~h}$ prior to the terminal seizure; $\mathbf{c}$ CXR $4 \mathrm{~h}$ prior to the terminal seizure; $\mathbf{d}$ CXR $12 \mathrm{~h}$ after the terminal seizure: $\mathbf{e} H C T 11 \mathrm{~h}$ prior to the terminal seizure; $\mathbf{f} \mathrm{HCT} 9 \mathrm{~h}$ after the terminal seizure without acute changes; $\mathbf{g}$ HCT $12 \mathrm{~h}$ after the terminal seizure with diffuse cerebral edema 


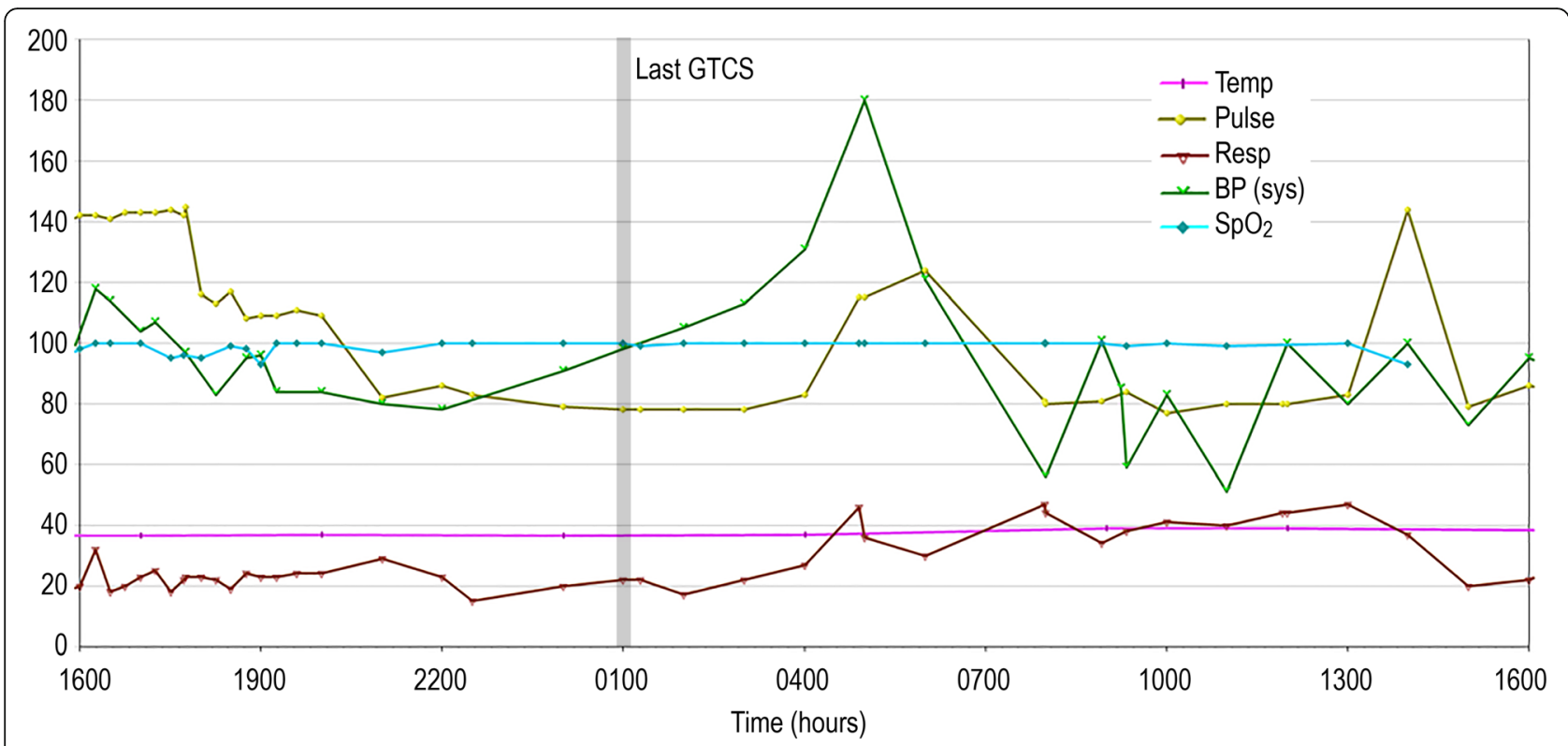

Fig. 3 Temporal illustration of periictal vital signs including heart rate, blood pressure, and body temperature and oxygen saturation over the course of $24 \mathrm{~h}$. The possible terminal GTCS and PGES occurred at the time of 1:00 and brain death occurred at the time of 12:00

\section{Discussion}

SUDEP is defined as sudden unexpected death (non-traumatic and non-drowning) in an individual with epilepsy with or without evidence of a terminal seizure and excluding documented status epilepticus (seizure duration $>30$ min or seizures without recovery in between) and which investigation and postmortem examination, including toxicology, do not reveal a cause of death other than epilepsy [9]. SUDEP has been observed without preceding epileptic seizures during video-EEG monitoring, reflecting the heterogeneity of SUDEP symptomatology [10]. This case is conceptually not considered a SUDEP case, in which the patient died hours after the possible terminal GTCS, likely delayed by the mechanical ventilation. Additionally, she did not have a history of epilepsy prior to this hospital admission, and her death was likely caused by acute symptomatic seizures. Nevertheless, acute symptomatic seizures share the similar clinical pathophysiological characteristics to that of epileptic seizures. Given that SUDEP has not been recorded under the respiratory and oxygen monitoring, this unique case may provide important insights into the mechanisms of SUDEP. Additionally, SUDEP has also been reported in patients with new onset of seizures [11].

There were likely two seizures prior to the likely terminal GTCS. One was un-witnessed, but suspected based on oxygen desaturation and urinary incontinence, and the other was witnessed by an EEG technologist prior to EEG being hooked up fully. Death occurred after the likely terminal GTCS when the patient was undergoing mechanical ventilation, presumably under the best case-scenario for SUDEP prevention after a seizure. However, the mechanical ventilation only delayed the death for $12 \mathrm{~h}$. Meanwhile, PGES was observed after the GTCS in the presence of stable vital signs, including heart rate, blood pressure, body temperature and oxygen saturation at the time of seizure termination, suggesting the underlying pathogenesis of PGES might be related to seizure-induced diffuse cerebral suppression and is independent of cerebral oxygen saturation [12]. The causes of seizures were not clear. It was possible that CNS inflammation, infection and electrolyte disturbances might be contributory, but evidences supporting these causes were lacking. The cause of death in this case was likely due to diffuse cerebral edema and herniation as supported by the findings of head CT and autopsy results. Although the factors leading to diffuse cerebral edema were unclear, cerebral hypoxic injury related to seizureinduced autonomic dysfunction and hypotension $(<60$ $\mathrm{mmHg}$ ) might be the contributing factors (Fig. 3) [13]. Seizure induced central and peripheral apneas as commonly suspected mechanisms of SUDEP were unlikely the direct causes of death in this case, given that the patient was intubated and mechanically ventilated. There was no fatal cardiac arrhythmia based on the EKG recording. Consecutive chest X-ray did not show significant neurogenic pulmonary edema (Fig. 2).

\section{Conclusions}

To our knowledge, this is one of the first cases reporting seizure-induced sudden unexpected death while the victim's airway was protected by intubation and mechanical 
ventilation. Although the cause of the death was not clear, cerebral edema secondary to autonomic dysfunction, hypotension and hypoxemia was likely the cause in this case. Fatal cardiac arrhythmia, central and obstructive apneas were not the direct causes of the death. A majority of SUDEP occurs during sleep, in bed and in the prone position, with most cases being unwitnessed [7]. These patients often live alone or are unsupervised during the sleep period. These common circumstances of SUDEP have highlighted the importance of periictal supervision to the prevention of SUDEP. In the case presented here, periictal airway protection was not effective in preventing the seizure related death, which underscores the challenges of SUDEP prevention in witnessed cases. This case also raises the question if witnessed SUDEP might have a different pathogenesis from unwitnessed SUDEP.

\section{Abbreviations}

EEG: Electroencephalography; GTCS: Generalized tonic clonic seizures; NCS: Nonconvulsive seizures; PGES: Postictal generalized EEG suppression; SUDEP: Sudden unexpected death in epilepsy

\section{Acknowledgements}

N/A.

\section{Authors' contributions}

RCM: Collected data and wrote the manuscript; NPI: Revised the manuscript for intellectual context; SW, XL, and SR: Reviewed literature and the manuscript for intellectual context; JXT: conceived the concept and revised the manuscript. The authors read and approved the final manuscript.

\section{Authors' information}

Dr. Martinez: Assistant professor in the department of neurology at the Emory University, USA.

Dr. Issa: Assistant professor in the department of neurology at the University of Chicago, USA.

Dr. Wu: Assistant professor in the department of neurology at the University of Chicago, USA.

Dr. Liu: Associate professor in the department of neurology at Wuhan University, China.

Dr. Rose: Assistant professor in the department of neurology at the

University of Chicago, USA.

Dr. Tao: Associate professor in the department of neurology at the University of Chicago, USA.

\section{Funding}

The study was not supported by any funding.

\section{Availability of data and materials}

Supporting data are available upon request.

\section{Ethics approval and consent to participate}

The study was approved by Intuitional Review Board of the University of Chicago (IRB18-093): Consent to participate was not required by the IRB due to minimal risk in deceased subjects.

\section{Consent for publication}

All authors agreed for the publication of this study.

\section{Competing interests}

Authors have no conflicts of interest.

\section{Author details}

'Adult Epilepsy Center, Department of Neurology, The University of Chicago, 5841 South Maryland Ave. MC2030, Chicago, IL 60637, USA. ${ }^{2}$ Department of
Neurology, Zhongnan Hospital, Wuhan University, Wuhan, Hubei Province, P. R. China.

Received: 17 July 2020 Accepted: 28 October 2020

Published online: 30 November 2020

References

1. Shorvon S, Tomson T. Sudden unexpected death in epilepsy. Lancet. 2011; 378:2028-38.

2. Walczak TS, Leppik IE, D'Amelio M, Rarick J, So E, Ahman P, et al. Incidence and risk factors in sudden unexpected death in epilepsy: a prospective cohort study. Neurology. 2001;56:519-25.

3. Langan $Y$, Nashef L, Sander JW. Case-control study of SUDEP. Neurology. 2005;64:1131-3.

4. Sveinsson O, Andersson T, Carlsson S, Tomson T. Circumstances of SUDEP: a nationwide population-based case series. Epilepsia. 2018;59:1074-82.

5. Hesdorffer DC, Tomson T, Benn E, Sander JW, Nilsson L, Langan Y, et al. Epidemiology ICo, subcommission on M. Combined analysis of risk factors for SUDEP. Epilepsia. 2011:52:1150-9.

6. Ryvlin P, Rheims S, Lhatoo SD. Risks and predictive biomarkers of sudden unexpected death in epilepsy patient. Curr Opin Neurol. 2019;32:205-12.

7. Liebenthal JA, Wu S, Rose S, Ebersole JS, Tao JX. Association of prone position with sudden unexpected death in epilepsy. Neurology. 2015;84: 703-9.

8. Ryvlin P, Nashef L, Lhatoo SD, Bateman LM, Bird J, Bleasel A, et al. Incidence and mechanisms of cardiorespiratory arrests in epilepsy monitoring units (MORTEMUS): a retrospective study. Lancet Neurol. 2013;12:966-77.

9. Nashef $L$, So EL, Ryvlin P, Tomson T. Unifying the definitions of sudden unexpected death in epilepsy. Epilepsia. 2012;53:227-33.

10. Lhatoo SD, Nei M, Raghavan M, Sperling M, Zonjy B, Lacuey N, et al. Nonseizure SUDEP: sudden unexpected death in epilepsy without preceding epileptic seizures. Epilepsia. 2016:57:1161-8.

11. Hebel JM, Surges R, Stodieck SRG, Lanz M. SUDEP following the second seizure in new-onset epilepsy due to limbic encephalitis. Seizure. 2018;62: 124-6.

12. Bauer PR, Thijs RD, Lamberts RJ, Velis DN, Visser GH, Tolner EA, et al. Dynamics of convulsive seizure termination and postictal generalized EEG suppression. Brain. 2017;140:655-68.

13. Bozorgi A, Chung S, Kaffashi F, Loparo KA, Sahoo S, Zhang GQ, et al. Significant postictal hypotension: expanding the spectrum of seizureinduced autonomic dysregulation. Epilepsia. 2013;54:e127-30. 